\title{
The influence of crystallographic orientation on the wetting of silicon on quartz single crystals
}

\author{
Daniel Weiß • Tim Gebensleben • Lisa Diestel • \\ Lukas Alphei • Verena Becker · Jörg August Becker
}

Received: 7 September 2010/ Accepted: 31 December 2010/Published online: 15 January 2011

(C) The Author(s) 2011. This article is published with open access at Springerlink.com

\begin{abstract}
Dynamic hexagonal spreading patterns of small silicon droplets on the basal plane (001) of quartz were observed by video microscopy. A detailed analysis of the hexagonal triple line demonstrates that the patterns show slight chiral distortions that can be attributed to the screw axis of the substrate crystal. This article reveals the detailed influence of crystal symmetry on the anisotropy of reactive wetting. In this context, a first discussion about the interplay of wetting and etching of a crystal is provided.
\end{abstract}

\section{Introduction}

Wetting and interface reactions are important phenomena in nature [1] and of great interest for materials engineering [2-10]. The wetting of oxide crystals, by metals in particular, is important for metal ceramic joints [11-13] and is also an interesting physicochemical problem since it involves wetting in combination with reaction dynamics. In industrial crystal growth $[14,15]$ it is observed that a quartz crucible which is wetted by a silicon melt is slowly corroded according to the reaction

$\mathrm{Si}$ (liquid) $+\mathrm{SiO}_{2}$ (solid) $\rightarrow 2 \mathrm{SiO}$ (gas).

Even for this simple reaction, the interplay between wetting and chemical reaction is not well understood.

This work is dedicated to Prof. Dr. Hansgeorg Schnöckel on the occasion of his 70th birthday.

D. Weiß · T. Gebensleben - L. Diestel · L. Alphei

V. Becker · J. A. Becker $(\square)$

Institut für Physikalische Chemie und Elektrochemie,

Gottfried-Wilhelm-Leibniz Universität Hannover,

Callinstr. 3A, 30167 Hannover, Germany

e-mail: joerg-august.becker@pci-uni-hannover.de
Moreover a detailed physical-chemical understanding of reactive wetting between liquid silicon melt and $\mathrm{SiO}_{2}$ is important for the advanced crystal growth of silicon [16-27]. Several wetting experiments with silicon melt on fused quartz have been reported in the literature [25-27], and complex dynamical spreading effects have been observed. The origin of these effects remains elusive. Furthermore, little is known about the chemical interaction between the silicon melt and the solid silicon dioxide. Previous investigations used amorphous substrates that can provide isotropic wetting data. Single crystal substrates, however, can reveal anisotropic effects in reactive wetting resulting from the anisotropy of the crystal structure as was recently demonstrated using a sessile drop experiment for a silicon droplet on a sapphire substrate [28]. Quartz crystal substrates are especially interesting because their structure is based on interconnected parallel hexagonal double helices of $-\mathrm{Si}-\mathrm{O}-\mathrm{Si}-$ chains with the same screw direction. This is illustrated in Fig. 1 for the high temperature modification of quartz ( $\beta$-quartz). Depending on the handedness of the helices there are two types of crystals, left and right handed quartz. The screw direction of the $-\mathrm{Si}-\mathrm{O}-\mathrm{Si}-$ helices might influence the wetting pattern and this is an interesting question to address experimentally. Investigations in this field are greatly facilitated by the existing knowledge about silicon melts, the crystal structure and phase transitions of quartz and the fact that the thermochemical data of the latter are known with high precision $[29,30]$. It should be mentioned that wetting phenomena of molten metals and semiconductors have conventionally been studied on polycrystalline substrates; the effect of crystallographic plane was not yet sufficiently discussed. We believe that basic scientific information can be obtained through this study, when single crystal substrates with well-defined crystallographic 
(a)

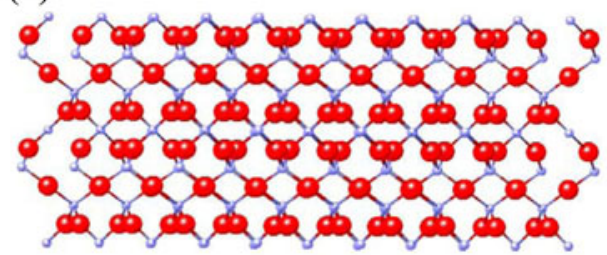

(c)

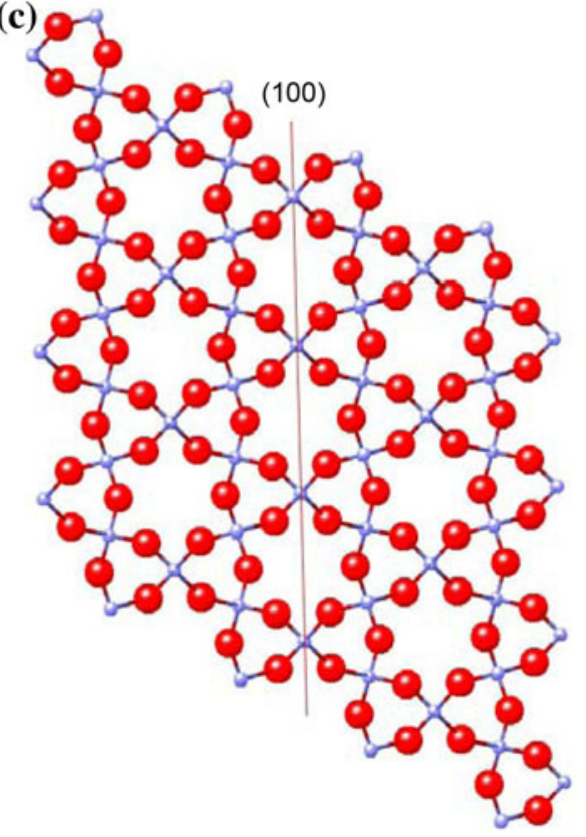

(b)

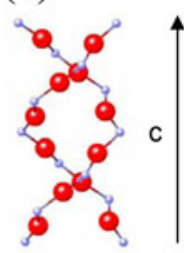

(d)

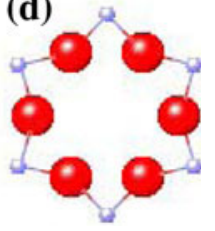

Fig. 1 Bonding structure of a right handed $\beta$-quartz which is composed by interconnected chiral hexagonal $-\mathrm{Si}-\mathrm{O}-\mathrm{Si}-$ double helices of right handedness (small light spheres: $\mathrm{Si}$, large dark spheres: O). (a) View of the crystal along $\langle 100\rangle$, (b) single right handed hexagonal $-\mathrm{Si}-\mathrm{O}-\mathrm{Si}-$ double helix winding upwards along the $c$-axis $\langle 001\rangle$, (c) top view on the basal plane of the $\beta$-quartz bonding structure. In this two dimensional projection there seem to be mirror planes as, e.g., (100), however, in three dimensions these mirror symmetries are broken because of the $-\mathrm{Si}-\mathrm{O}-\mathrm{Si}-\mathrm{screws}$, (d) top view along $\langle 00-1\rangle$ on a single hexagonal double helix. In the left handed $\beta$-quartz crystal the screw direction of the hexagonal double helices is simply reversed i.e., left handed

orientations are used. In this way one can investigate how the chemical interaction between the atoms in the melt and substrate surface is influenced by the bond structures in the crystal.

A silicon droplet that is placed on the surface of a heated fused $\mathrm{SiO}_{2}$ substrate frequently moves around the surface, but if it rests it shows shape oscillations that have been related to the dynamics of the reactive wetting. There are several models that try to explain these observations, e.g., through $\mathrm{SiO}$ gas bubble formation or Marangoni effects [7, 25-27, 31, 32], but much remains unclear. Hence, new experimental investigations on polished and crystallographic well oriented quartz crystal substrates are desirable, and the goal of this study.

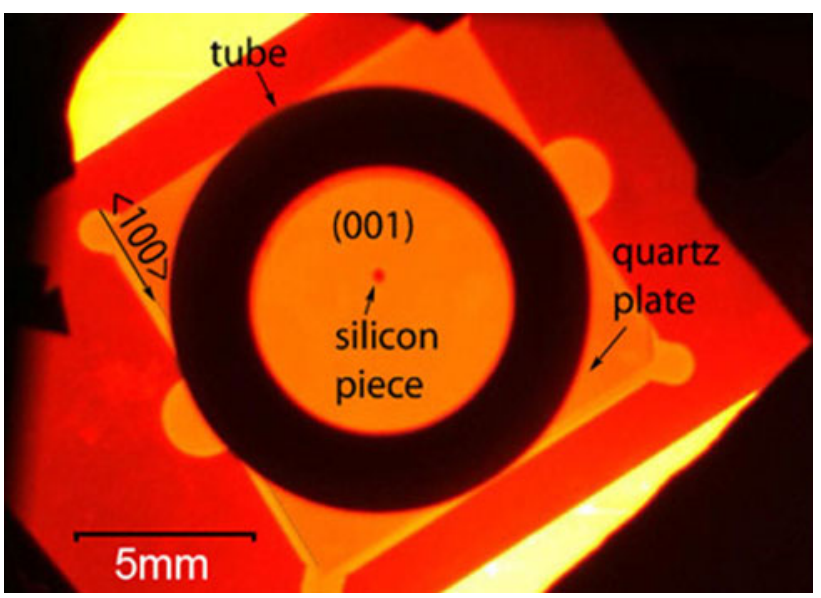

Fig. 2 Overview of the experimental setup through the microscope that is looking (under low magnification) into the the top sapphire window of the vacuum chamber. A tiny piece of silicon is placed on a polished quartz substrate $\left(10 \times 10 \times 0.5 \mathrm{~mm}^{3}\right)$, melted and then supercooled. The tube provides uniform lighting conditions around the droplet which is crucial for a detailed analysis of the contour line of the melt droplet. The tube can be removed to get a side view on the droplet by use of a second microscope looking through the side window of the apparatus

\section{Experiment}

In order to investigate these effects under well-defined conditions we used a sessile drop apparatus similar to that used in our experiments with sapphire crystals [28]. A top view of the experimental setup in the vacuum chamber is shown in Fig. 2. The measurements took place under ultra high vacuum conditions with a pressure of $10^{-7} \mathrm{~Pa}$ before heating and typically $10^{-4} \mathrm{~Pa}$ under reaction conditions. In our first experiment, we cut substrates from a natural quartz crystal parallel to its basal plane, whereas in later experiments we used synthetic crystals. ${ }^{1}$ In both cases we observed very similar shape fluctuations with typical frequencies of several Hertz at the melting point of $1687 \mathrm{~K}$. In order to slow down these oscillations the silicon droplets were supercooled, typically to $20-80 \mathrm{~K}$ below the melting point, at which point the wetting dynamics can be easily recorded by means of a video microscope. ${ }^{2}$ The droplet was observed through a sapphire window from above or from one side, and the temperature of the droplet was determined by a 2 -colour pyrometer.

\footnotetext{
1 The natural crystals stem from Minas Gerais in Brasil, while the synthetic ones were hydrothermally grown and supplied by MTI and Roditi Corporation.

2 An Olympus SZX2 Microscope and a Pike video camera of Allied Corporation were used and the recording and analysis was accomplished by the LABVIEW software in combination with MATLAB.
} 


\section{Results and discussions}

In contrast to previous studies on fused silica substrates [25-27], we did not observe any gas bubble formation from our small droplets on the polished surfaces of the single crystal substrates. A possible reason for this will be discussed at the end of this article and the details will be analyzed in a later publication that concentrates on the reaction mechanisms.

The experiments permitted us to record largely spread regular anisotropic wetting patterns especially when using small droplets (about $200 \mu \mathrm{m}$ diameter) on the basal plane, i.e., c-face (001) of quartz at $1610 \mathrm{~K}$. In Fig. 3 a sequence of wetting patterns during one single spreading of the droplet is shown. The observation direction is perpendicular to the quartz surface. The corresponding side view experiment on the basal plane of a crystalline substrate (Fig. 4) shows the typical spreading/contraction sequences that have been reported for larger melt droplets on flat fused quartz substrates [25-27]. However, it is difficult to reveal the anisotropic effects in the side view (see Fig. 4). For this reason all experiments were conducted under top view observation. The spreading sequence of a silicon droplet on a fused quartz substrate from top view observation is shown in Fig. 5. One can clearly observe that the spreading pattern is almost perfect circular as expected for the non crystalline substrate. It should be mentioned that impurity segregation was observed by optical microscopy at the surface of the natural quartz after the experiments, while such an effect was not seen for the synthetic quartz. The spreading patterns and its dynamics are very similar for both the samples and hence impurity segregation seems to have only a small effect.

\section{Motion of triple line}

The sequence of wetting patterns in Fig. 3a-e shows the first phase, lasting for about $3 \mathrm{~s}$, when the droplet slowly flattens with approximately constant triple line velocity and assumes at the end the shape of a hexagon with rounded (capped) edges (Fig. 3e). The diameter of the droplet increases almost by a factor of two. After that, in the second phase, it suddenly retracts to its original circular shape within about $100 \mu$ s (Fig. 3f). During the slow spreading phase, the contact angle decreases from an initial value of about $\theta=90^{\circ}$ to typically $\theta=50^{\circ}$, as can be observed in the side view experiments presented in Fig. 4. The contact angle can also be estimated from the
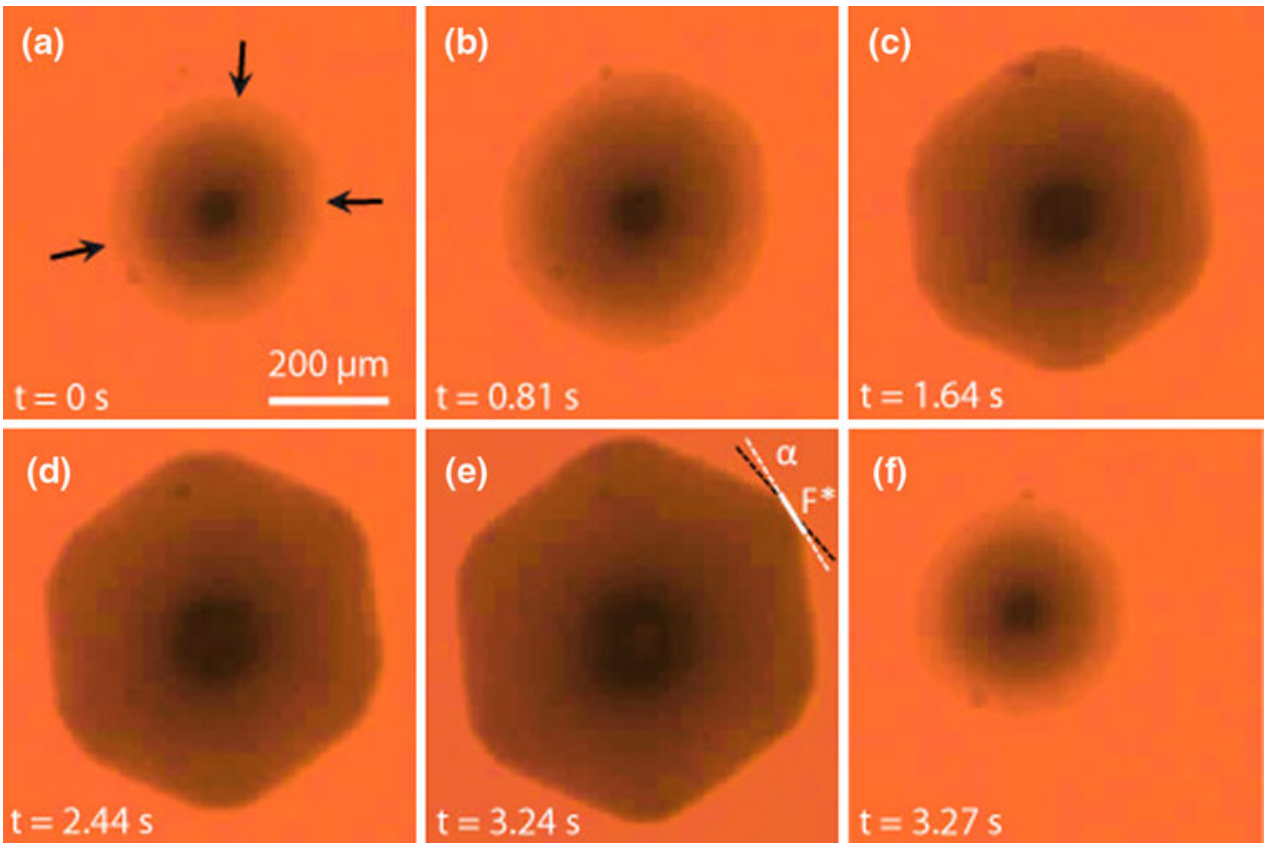

Fig. 3 Spreading of a melt droplet on the basal plane of a right handed quartz. Top view on a supercooled droplet in the vacuum chamber using a high resolution CCD Camera ${ }^{2}$. The nearly round droplet (a) spreads slowly (b-e) and is thereby distorted into a hexagonal pattern. Each of the rounded edges of the hexagonal pattern exhibits a small flattened region i.e., an additional facet $\mathrm{F}^{*}$. Schematic illustrations in (e): white dashed line: direction of small facet $\mathrm{F}^{*}$, black dashed line $\langle 210\rangle$ direction in crystal. There is a small angle $\alpha$ between these directions. The spread droplet suddenly retracts to its round shape (f) and a new spreading cycle begins. Note that the droplet acts like a spherical mirror. The dark spot in the center of the pattern is a reflection of the upper tube end (see Fig. 1), while the light colored band around the contracted droplet is a reflection of the quartz plate. When the droplet flattens during spreading the reflection of the quartz substrate disappears. Black arrows indicate the triple line in (a) 

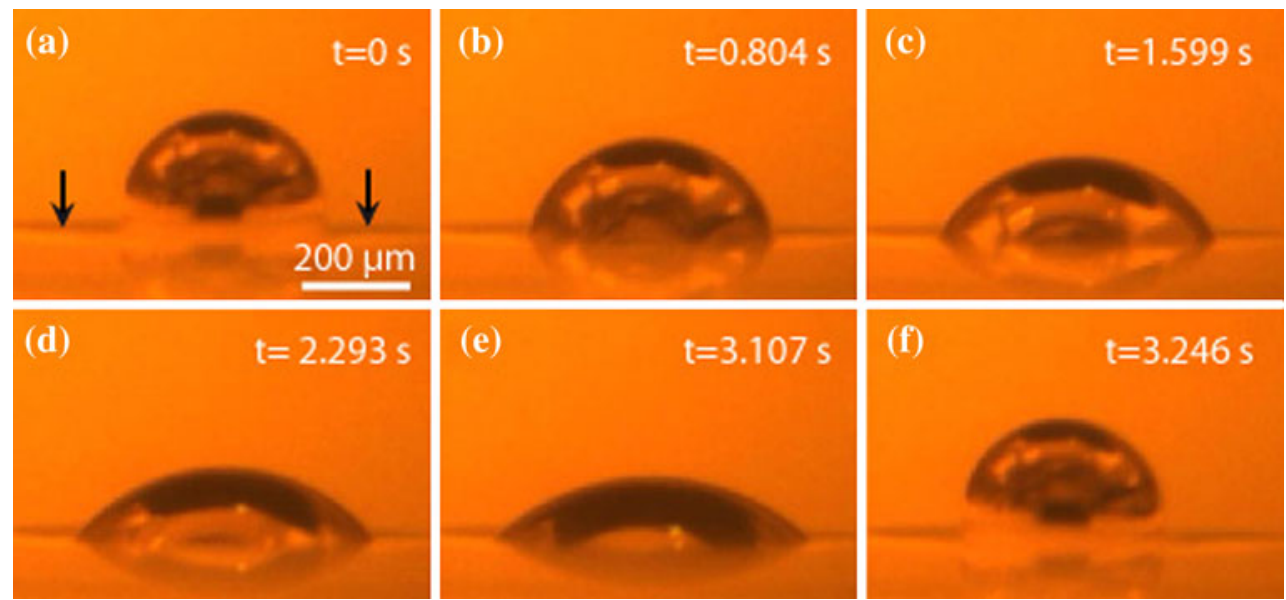

Fig. 4 Spreading of a melt droplet on the basal plane of a right handed quartz. Side view on a droplet under similar conditions as in Fig. 3. The droplet appears to be perfectly spherical and the hexagonal spreading patterns seen by the top view cannot be discovered. The structures on the droplet surface result from optical reflections of the heat shields. The contact angle changes from approximately $90^{\circ}$ (a) to $50^{\circ}$ (e) and with the retraction of the droplet it achieves its initial value again (f). Black arrows in (a) indicate the surface of the quartz substrate, the shadow on the quartz surface results from the shallow depression which is etched by the droplet
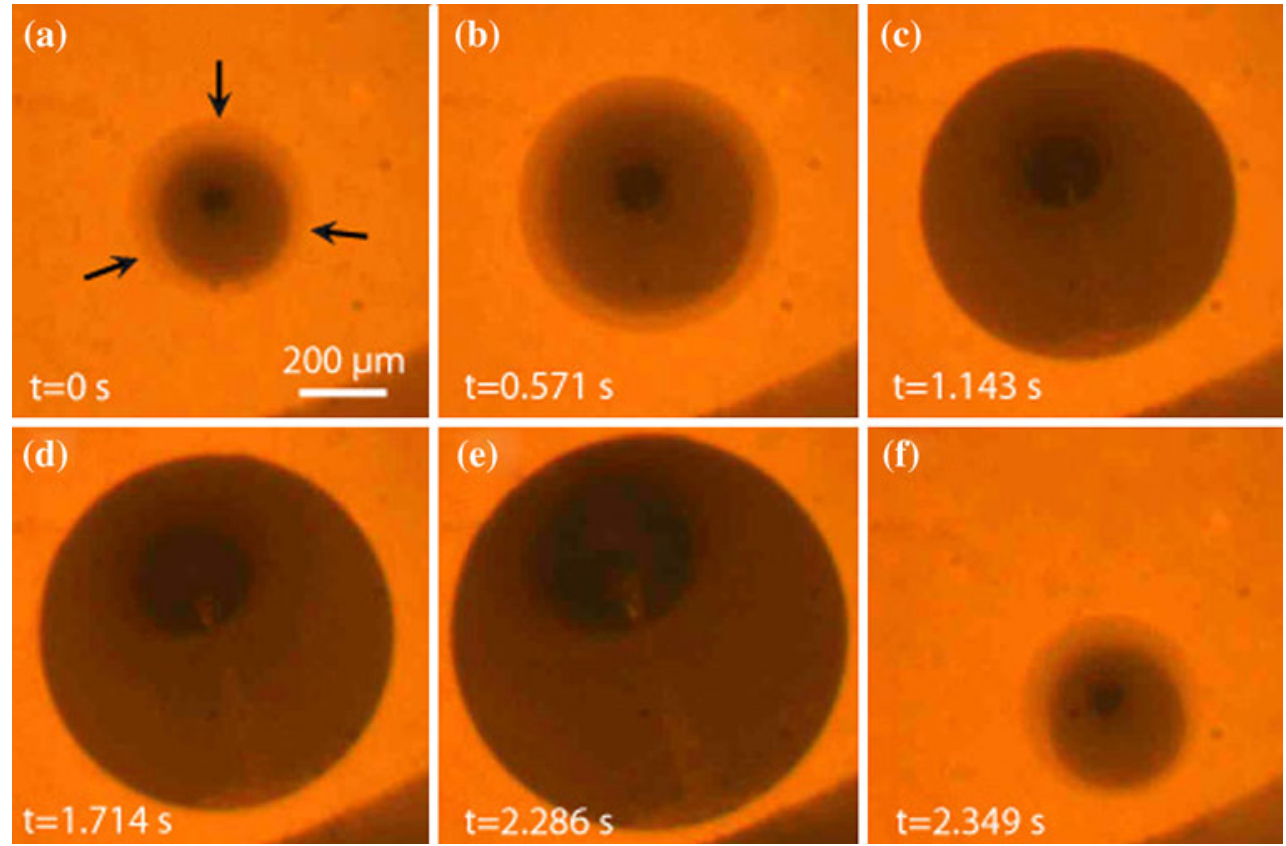

Fig. 5 Spreading of droplet on a fused (amorphous) quartz substrate. Top view on a droplet on a fused silica substrate under similar conditions as in Fig. 3. During spreading the droplet keeps an almost perfect circular shape. Black arrows in (a) show the triple line

weight ${ }^{3}$ of the droplet, the density of liquid silicon, and the diameter of the spreading pattern. The results are in good agreement with the observed contact angle from side view experiments. After the fast contraction sequence, a new cycle of wetting and dewetting begins. By supercooling the

\footnotetext{
3 The ultra micro balance SE2 from Sartorius was used to measure the weight of the silicon pieces with an accuracy of $0.1 \mu \mathrm{g}$.
}

melt one can observe very large spreading patterns and slow spreading processes up to $5 \mathrm{~s}$, providing very detailed pictures of the triple line geometry. Nevertheless, rapid but much smaller wetting pattern formation takes place at the melting point of silicon and above. The formation of hexagonal patterns is hence a general feature of the reactive wetting of the quartz basal plane (001) by small silicon droplets. 
By keeping the substrate strictly horizontal large and regular spreading modes on flat EPI-polished quartz substrates with crystal orientations better than $0.2^{\circ}$ can be analyzed. After melting and immediate supercooling to typically $30 \mathrm{~K}$ below the melting point, the droplet slowly etches a shallow cup into the substrate and confines itself, and very regular spreading patterns can be observed, recorded, and evaluated.

\section{Geometry of the triple line}

The shape of the spreading pattern on the basal crystal plane of quartz indicates that the reactive wetting is driven by anisotropic thermodynamic forces. Further, microscopic investigations have shown that the surface of the expanded droplet exhibits six hexagonally arranged depressions. A theoretical investigation applying an approach developed by de Gennes [1] shows that their existence is necessary to keep the mean surface curvature at a constant value according to the Young-Laplace equation [1].

At room temperature, quartz crystals have a trigonal structure ( $\alpha$-quartz), whereas at the elevated temperature of the experiment the quartz transforms to the $\beta$-phase [14] $\left(T_{\text {trans }}=846 \mathrm{~K}\right)$ which belongs to the hexagonal-trapezoidal crystal class. The observation of a hexagonal symmetry of the wetting patterns is therefore consistent with the crystal's symmetry class. At first glance it may seem that the wetting pattern (Fig. 3e) possesses six mirror planes, however, this can be only approximately correct because they are not symmetry elements of the hexagonaltrapezoidal crystal class of the $\beta$-quartz. Instead of such mirror planes in higher symmetric hexagonal crystal classes, this class only possesses a screw axis which is microscopically related to the existence of $-\mathrm{Si}-\mathrm{O}-\mathrm{Si}-$ double helices [29] as illustrated in Fig. 1. This causes the well-known chirality of $\alpha$ - and $\beta$-quartz and gives rise to the optical activity of both the modifications. In addition to optical activity, chemical effects can result from the chirality of quartz; e.g., the preferred adsorption of one of the stereo isomers of a chiral molecule [33] or the enantioselective catalysis of organic reactions by means of metal layers on fine quartz particles [34]. Reactive wetting can also be affected by the chirality of the crystal substrate, and thus a search was undertaken for some distortion of the apparent mirror symmetry of the wetting pattern. It should be mentioned that the screw direction of quartz crystals can be determined chemically by etching, e.g., one of its prism faces by an acid [35]. After such a corrosion experiment one can observe specific anisotropic etch figures that reflect the handedness of the crystal.

A crucial feature of the wetting pattern are the small facets which delimit the edges of the hexagonal pattern, as depicted for example in Fig. $3 \mathrm{e}$ as $\mathrm{F}^{*}$ by the thick white line. A first indication of chiral effects in the wetting pattern is given by the orientation of these small facets. The direction of the facet $\mathrm{F}^{*}$ can be extended by the white dotted line and it seems that this direction does not coincide with the $\langle 210\rangle$ direction that is shown schematically by the black dotted line. There is a small angle $\alpha$ between these directions. Without chirality this angle should vanish. In order to quantify this observation we have developed an evaluation procedure that can supply us with the angle $\alpha$ automatically.

Details of the triple line shape

The contour lines of the hexagonally spread droplet patterns were extracted by a small computer program directly from the video microscope pictures. A typical contour line of a maximally spread droplet on a right quartz substrate is shown in Fig. 6. As a first step, the deviation $d(\phi)$ of the contour line from a circle with radius $\langle r\rangle$ that gives a best fit to the contour line is obtained by subtraction of $\langle r\rangle$ from $r(\phi)$. The anisotropic spreading effect is then quantified by calculating the relative variation $D(\phi)=d(\phi) /\langle r\rangle$. Then, the function $D(\phi)$ is plotted. Typical plots are shown for a right and a left handed quartz substrate in Fig. 7, and asymmetric peak shapes are evident for both the quartz types. There seems to be a simple rule: the peaks in these

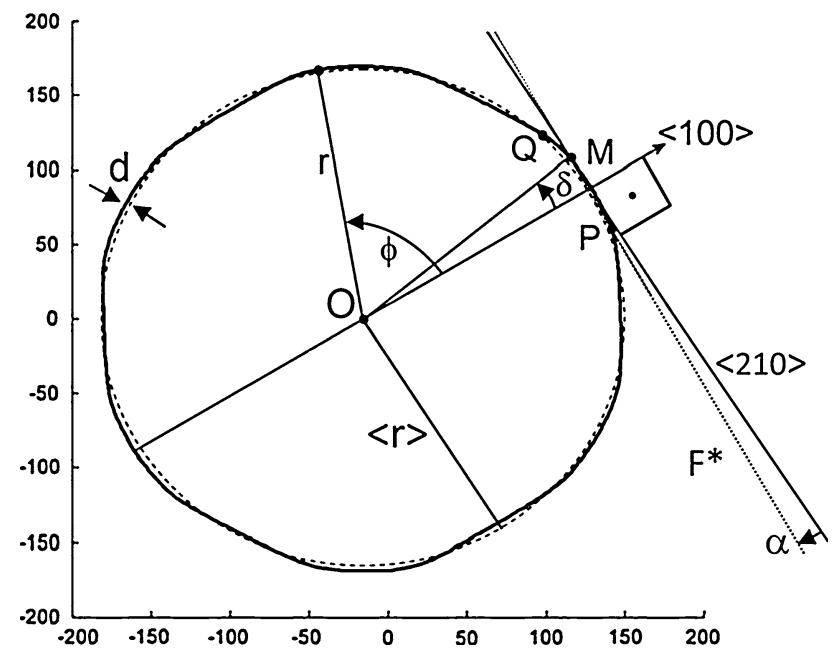

Fig. 6 Definition of geometric quantities used to analyze effects of chirality in the wetting patterns, as explained in the text. A contour line is plotted (thick full line, here: right quartz substrate) and evaluated: In a first step the center of gravity $\mathrm{O}$ of the experimental contour line is determined and a circle with the average radius $\langle r\rangle$ is drawn around it (dashed line). Then the angle $\phi$ of each contour line point with respect to the substrates $\langle 100\rangle$ direction is determined. Each point on the contour line is then specified by its distance $r$ from the center $\mathrm{O}$ and the angle $\phi$. The direction of the facet $\mathrm{F}^{*}$ can be quantified by use of the angle $\alpha$ with respect to the crystal lattice directions $\langle 210\rangle$ 

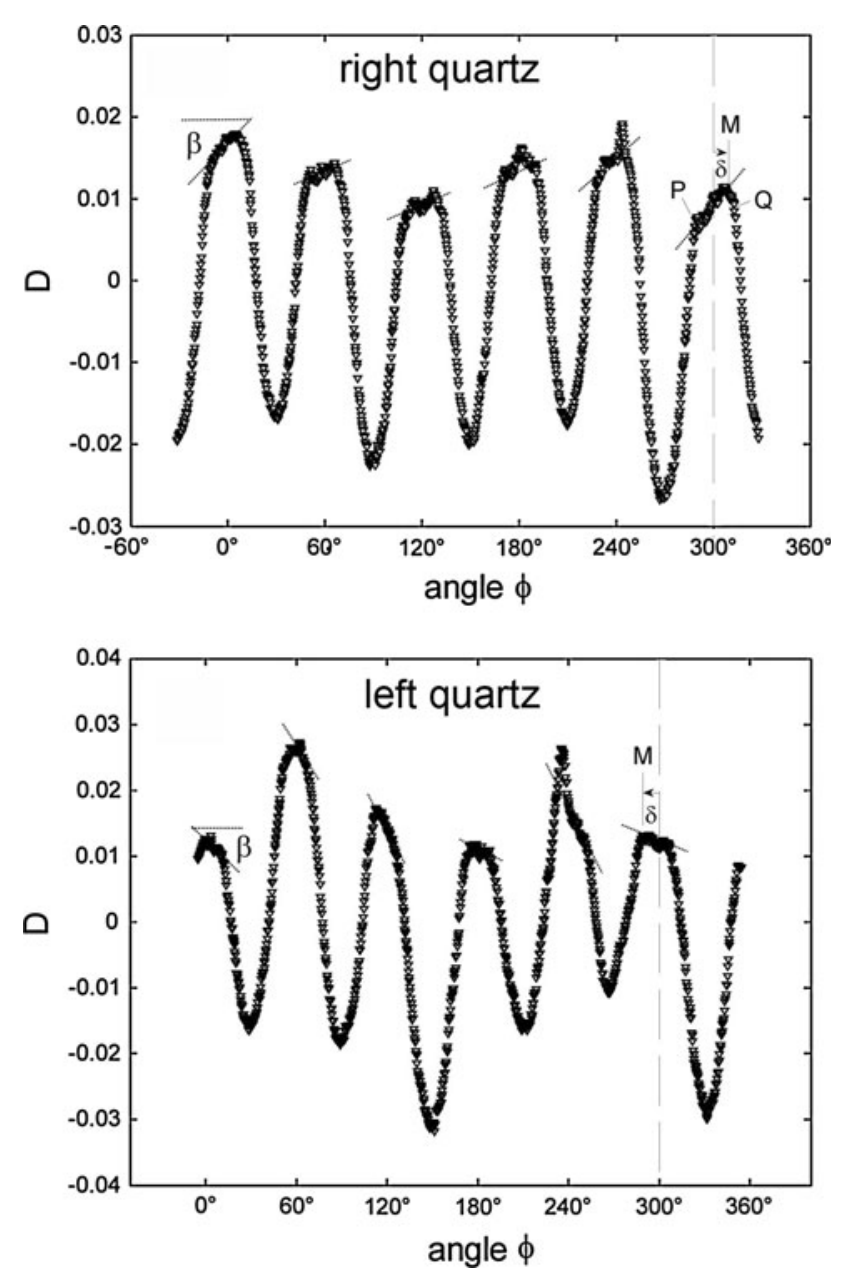

Fig. 7 Plot of relative asymmetric triple line distortion $D(\phi)$ for a right handed quartz substrate and a left handed quartz as explained in the text. The peaks are clearly asymmetric. The rule is for right quartz, the maxima $M$ are found on the right side of the corresponding lattice directions (e.g., dashed line at $300^{\circ}$ ) whereas they lie on the left side for left quartz. From these slopes one determines the angle $\alpha$ as shown in Fig. 6. The angle $\delta$ is the shift of the maximum radius at $M$ against the lattice directions with $\phi=0^{\circ}, 60^{\circ}$, and $120^{\circ}$ etc

plots exhibit flattened regions in the range of about $\pm 5^{\circ}$ around the positions $\phi=0^{\circ}, 60^{\circ}$, and $120^{\circ}$, etc., with positive slopes $\beta$ for right handed quartz and negative slopes $\beta$ for left handed quartz.

Following the contour line through the points $\mathrm{P}, \mathrm{M}$, and $\mathrm{Q}$ in the plots of Fig. 7, it becomes clear that the magnitude of the angle $\alpha$ is proportional to the corresponding slope $\beta$. Because the $\mathrm{F}^{*}$ facet has to be rotated by a negative angle to coincide with the $\langle 210\rangle$ direction, $\alpha$ is set to a negative value for the geometric situation in Fig. 7 (right quartz). The maxima $M$ of the peaks are therefore shifted by positive angles $\delta$ for right quartz with respect to the positions $\phi=0^{\circ}, 60^{\circ}$, and $120^{\circ}$ etc. The opposite rule is found for the left quartz substrates: positive $\alpha$ and negative $\beta$ values.
In order to quantify the chiral effect, the size of the average angles $\alpha_{\mathrm{P}}$ and $\delta_{\mathrm{P}}$ for each wetting pattern at the point of maximum extension are calculated along with the corresponding root mean square deviations $\operatorname{RMS}\left(\alpha_{\mathrm{P}}\right)$ and $\operatorname{RMS}\left(\delta_{\mathrm{P}}\right)$. In some cases the spreading pattern was still too irregular (e.g., due to defects on the substrates) and the RMS became larger than the average. Therefore, only selected patterns with $\operatorname{RMS}\left(\delta_{\mathrm{P}}\right)<\left|\delta_{\mathrm{P}}\right|$ and $\operatorname{RMS}\left(\alpha_{\mathrm{P}}\right)<\left|\alpha_{\mathrm{P}}\right|$ are shown in the histograms in Fig. 8 for $\delta_{\mathrm{P}}$ and Fig. 9 for $\alpha_{\mathrm{P}}$ which demonstrate the systematic influence of the crystal structure on the chirality of the reactive wetting pattern. The meaning and the signs of the two angles can be further visualized by fitting a handed dodecagon to the pattern which touches the contour line. Such dodecagons are schematically shown in the insets of Fig. 8.

Because of the sensitive influence of the crystal structure on the wetting patterns, a preliminary discussion about the relation between wetting and crystal reaction is presented. A more comprehensive examination will be explored in a future publication.

\section{Wetting and etching}

There is no overall faceted etch pattern found in the substrate which reflects the structure of the spreading pattern. A probable reason is that the center of the droplet slightly moves during the experiment, causing a complex superposition of hexagonally shaped etch lines. Sometimes very large spreading patterns can appear where the triple line has advanced by a nearly constant velocity over a fresh area of the originally flat substrate surface. If one cools down the sample after such events it is possible to find lines on the substrates (see Fig. 10) that correspond to the maximal advanced triple line positions; i.e., it follows a section of the hexagonal triple line of the droplet as illustrated. This is a very important observation that demonstrates that the hexagonal wetting pattern formation occurs also during the reactive wetting of a perfectly planar basal crystal plane. Hence the anisotropic motion of the triple line is not simply a result of varying local surface morphologies i.e., wetting angles due to a curved crystal structure. Further examinations by atomic force microscopy (Nanosurf EasyScan 2) reveal smooth step like etch structures with a step height of typically 100-200 nm (Fig. 11). No microfacets are observed along with this etch structures.

Such a step can be essentially understood by an advancing triple line which is locally etching solid within a lateral distance of only a few hundred nanometers. In investigations of silicon crystal growth from a melt in fused quartz crucibles [19-24, 26] oxygen is observed to dissolve from the quartz into the silicon melt. The oxygen solubility in the Si melt is very small $[14,15]$, but it is well known 
Fig. 8 Histogram for the angle $\delta_{\mathrm{P}}$ of a set of patterns as explained in the text. The sign of $\delta_{\mathrm{P}}$ reveals the chirality of the pattern and its absolute value measures the extension of the facet $F^{*}$
Fig. 9 Histograms for the average angle $\alpha_{\mathrm{p}}$. The average slopes $\beta$ shown in Fig. 7 translate into positive $\alpha_{\mathrm{p}}$ values for left handed quartz and negative ones for right handed quartz, as explained in the text
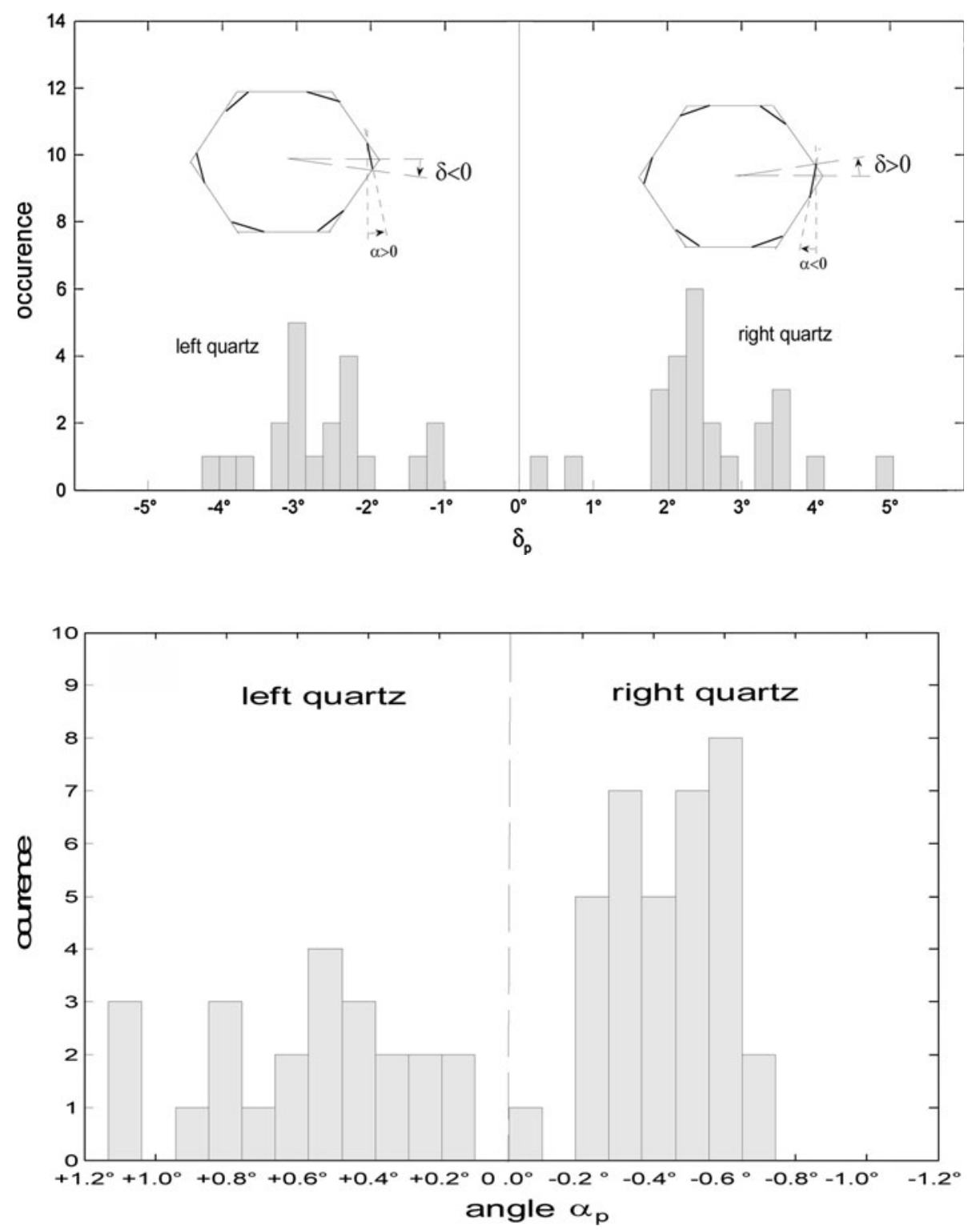

that oxygen can rapidly diffuse through the melt to the liquid surface and is then transferred as $\mathrm{SiO}$ molecules into the atmosphere (vacuum chamber). It is clear that the shortest paths for the oxygen diffusing from the solid/liquid to the liquid/vacuum interface will be found close to the triple line. Therefore, one expects high etch rates in the vicinity of the triple line. This process is further supported by an emission of $\mathrm{SiO}$ molecules, which increases proportional to the triple line circumference, as recently observed on-line by time resolved mass spectrometry in our study group. The details of these experiments will also be communicated in a future publication.

The velocity of the advancing triple line might be limited by the local etching rate at the step, which will depend on the orientation of the step in the crystal. The hexagonal shape of the triple line, i.e., the reaction front, could then reflect the anisotropic etching behavior of the crystal and explain the handedness of the spreading patterns. As illustrated in the model in Fig. 12a and b, the right handed $\beta$-quartz crystal exhibits besides its usual basal [e.g., (001)], prism [e.g., (100)] and pyramidal faces [e.g., (101) or (111)] so called trapezohedron faces as (511) while left quartz possesses instead a (6-11)-face. Such handed arrangements of crystal faces could be involved in the reactive wetting process and entail slight chiralities of the reactive wetting patterns. The formation of right and left handed etch patterns can be easily imagined by the use of basal-, prism- , and trapezohedron faces, i.e., handed negative crystals as presented in Fig. 12c and d. Such facetted etch patterns might be generated by crystal dissolution, 


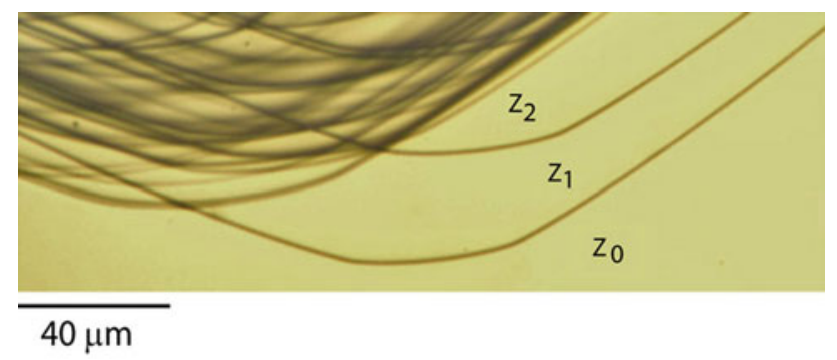

Fig. 10 Typical etch structures on the substrate after the experiment as explained in the text. The microscope picture (Jenavert microscope, Zeiss Jena) reveals lines that reflect the anisotropic behavior of reactive wetting of a planar (001) quartz surface. The height levels have been determined by AFM microscopy (Nanosurf EasyScan 2). The lines represent a shallow, smooth rise with a slope of roughly $10^{\circ}$. The areas between these lines are smooth and quite flat and form broad steps. The original substrate height level is denoted by $Z_{0}$, the level for the area etched by one spreading by $Z_{1}$, the level for two by $Z_{2}$. The step height between them is typically $100-200 \mathrm{~nm}$

provided that certain sets of such trapezohedron faces appear to be selectively resistant against etching with the silicon melt. Due to capillary action of the melt one does not expect sharp but rather rounded edges, as shown in the spreading patterns and the etch lines in Fig. 10.

It should be mentioned that (511) or (6-11) are only the best known low index trapezohedron faces that might be used to illustrate simple conventional etch patterns. There exists an infinite number of higher index faces that can come into play in the complex reactive wetting phenomenon on the basal plane. Moreover, in principle one can generate smooth rounded structures by a superposition of an infinite set of (high index) trapezohedron planes i.e., curved surfaces exhibiting chirality.

\section{Summary and conclusion}

The observation of small silicon melt droplets (of up to $500 \mu \mathrm{m}$ in diameter) on polished crystalline quartz has provided the means to study basic effects of reactive wetting. There are complex relations between wetting, capillary forces, and etching which results in spreading patterns that reflect details of the crystal structure. A detailed analysis of the geometry of the triple line demonstrates that the chirality of the crystal is reflected in a chirality of the wetting patterns. The advancing melt etches the crystal surface in the vicinity of the triple line and forms shallow hexagonal etch structures that might be explained by negative quartz crystal geometries. Such a negative crystal approach may be simplistic, but at least it demonstrates the importance of certain crystal faces for etching and hence reactive wetting, and can explain the handedness of the

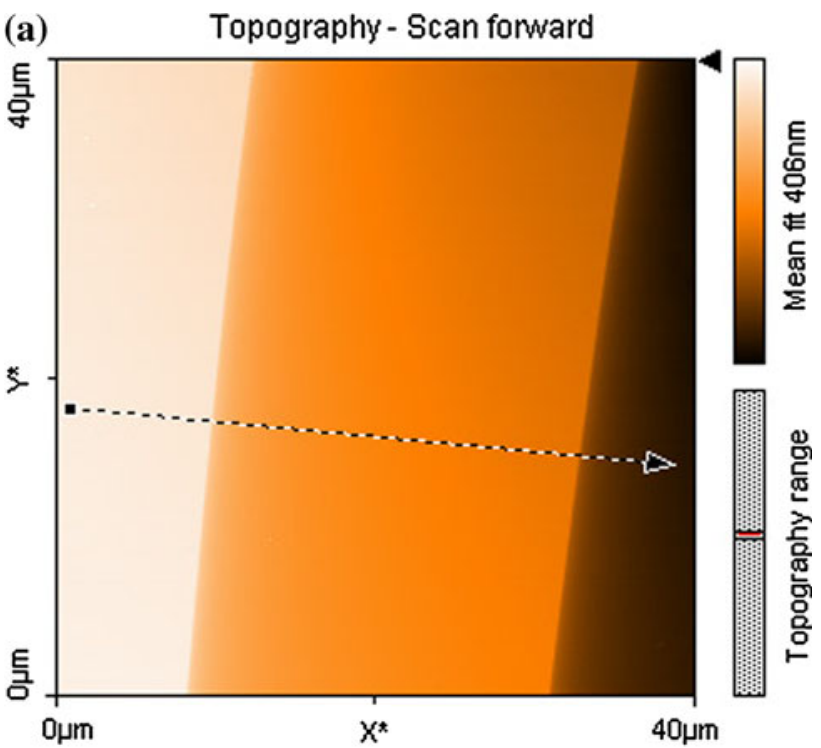

(b) Topography - Section

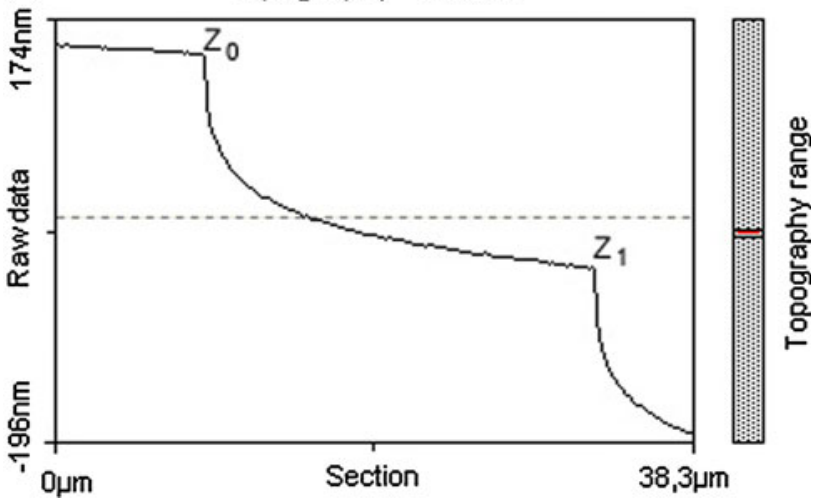

Fig. 11 AFM image of a typical step resulting from the spreading of the silicon droplet. (a) The image is depicted as color map (light shaded area means higher, dark shaded area means lower topographic level). On the left side (light region) is the original surface of the quartz substrate $\left(Z_{0}\right.$ in Fig. 10). The first step results from one single spreading of the melt. (b) Cross section along the arrow in (a), step height from $Z_{0}$ to $Z_{1}$ is $176 \mathrm{~nm}$ (Color figure online)

spreading patterns. The interaction between etching and local contact angle has not been investigated. These are complex but very interesting questions are to be targeted by further experimental and theoretical studies. Questions concerning anisotropic evolution of the triple line due to defects and their distribution along the crystal surface remain to be addressed. Upon completion of this manuscript we became aware of a very recent article [36] that discusses several fundamental issues concerning the anisotropy of triple lines. This study shows that the study of triple line geometry and its dynamics provides a wide and interesting research field because of its fundamental physical and chemical background and its possible impact on materials science. 

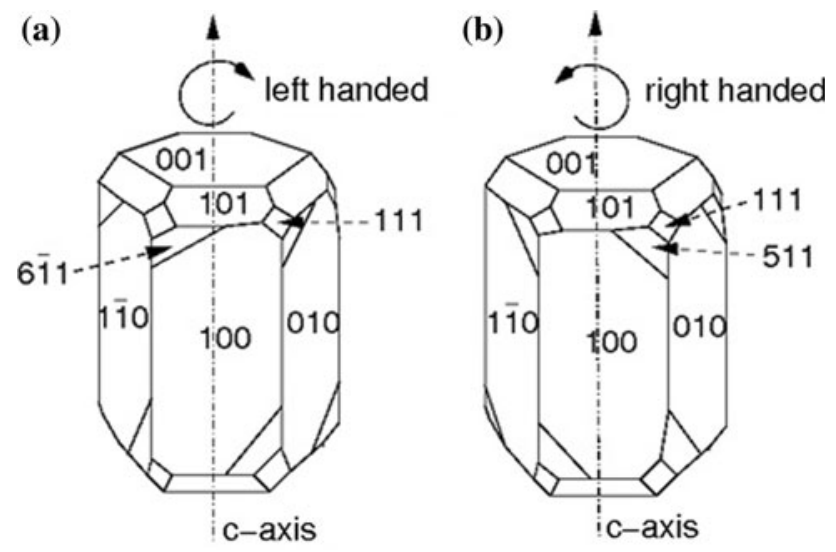

(c)
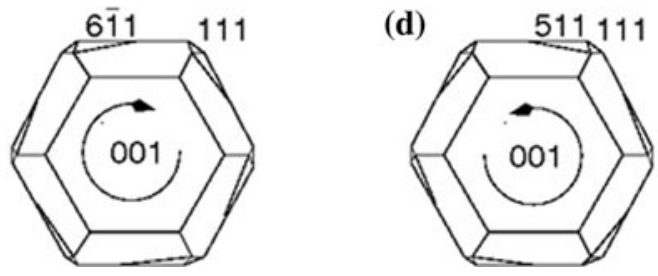

Fig. 12 Illustration of low index crystal faces of $\beta$-quartz. a, b The handedness is easily recognized by the position of the trapezohedron faces (511) for right handed quartz and (6-11) for left quartz. Illustration of a $\beta$-quartz crystal with prism (100), pyramidal (111) and trapezoidal (511), (6-11) faces for right and left handed quartz. c, d Top view on a negative crystal face arrangement illustrating the chirality of potential etch patterns by use of low index planes

Acknowledgements We thank PD Dr. M. Schmidbauer from the IKZ in Berlin for checking the orientation of two natural quartz crystals by X-ray diffraction.

Open Access This article is distributed under the terms of the Creative Commons Attribution Noncommercial License which permits any noncommercial use, distribution, and reproduction in any medium, provided the original author(s) and source are credited.

\section{References}

1. De Gennes PG, Brochard F, Quere D (2004) Capillarity and wetting phenomena: drops, bubbles, pearls, waves. Springer, New York

2. Dezellus O, Eustathopoulos N (2010) J Mater Sci 45:4256. doi:10.1007/s10853-009-4128-x
3. Mortensen A, Drevet B, Eustathopoulos N (1997) Scr Mater 36:645

4. Yost FG, Sackinger PA, O'Toole EJ (1998) Acta Mater 46:2329

5. Warren JA, Boettinger WJ, Roosen AR (1998) Acta Mater 46:3247

6. Hodaj F, Dezellus O, Barbier JN, Mortensen A, Eustathopoulos N (2007) J Mater Sci 42:8071. doi:10.1007/s10853-007-1915-0

7. Saiz E, Tomsia AP (2004) Nat Mater 3:903

8. Chatain D, Carter WC (2004) Nat Mater 3:843

9. Wynblatt P (2000) Acta Mater 48:4439

10. Chatain D (2008) Annu Rev Mater Res 38:45

11. Lewinsohn CA, Singh M, Loehman RE (2003) Advances in joining of ceramics. Wiley- $\mathrm{VCH}$, Weinheim

12. Jadoon AK (2008) J Mater Sci 43:1. doi:10.1007/s10853007-2217-2

13. Singh M, Shpargel TP, Asthana R (2008) J Mater Sci 43:23. doi:10.1007/s10853-007-1985-z

14. Carlberg T, King TB, Witt AF (1982) J Electrochem Soc 129:189

15. Evers J, Klüfers P, Staudigl R, Stallhofer P (2003) Angew Chem 115:5862

16. Kinney TA, Brown RA (1993) J Cryst Growth 132:551

17. Mukai K, Yuan Z, Nogi K, Hibiya T (2000) ISIJ Int 40:148

18. Cröll A, Lantzsch R, Kitanov S, Salk N, Szofran FR, Tegetmeier A (2003) Cryst Res Technol 38:669

19. Kim MC, Yi KW (1998) Met Mater 4:89

20. Togawa S, Huang X, Izunome K, Terashima K, Kimura S (1995) J Cryst Growth 148:70

21. Mühe A, Backofen R, Fainberg J, Müller G, Dornberger E, Tomzig E, von Ammon W (1999) J Cryst Growth 198(199):409

22. Hoshikawa K, Huang X (2000) Mater Sci Eng B72:73

23. Togawa S, Izunome K, Kawanishi S, Chung SI, Terashima K, Kimura S (1996) J Cryst Growth 165:362

24. Hirata H, Hoshikawa K (1990) J Cryst Growth 106:657

25. Fujii H, Yamamoto M, Hara S, Nogi K (1999) J Mater Sci 34:3165. doi:10.1023/A:1004673605025

26. Mukai K, Sako T, Yuan Z, Su Z (2000) Mat Trans JIM 41:639

27. Kanai H et al (2007) J Mater Sci 42:9529. doi:10.1007/s10853007-2090-z

28. Braun AM et al (2009) Z Phys Chem 223:329

29. Heaney PJ, Veblen DR (1991) Am Miner 76:1018

30. Richet P, Bottinga Y, Denielou L, Petitet JP, Tequi C (1982) Geochim Cosmochim Acta 46:2639

31. Hibiya T et al (1998) Philos Trans R Soc London Ser A 356:899

32. Rongy L, De Witt A (2007) J Eng Math 59:221

33. Bonner WA, Kavasaneck P, Martin FS, Flores JJ (1974) Science $186: 143$

34. Klabunovsky E, Smith GV, Zsigismond A (2006) Heterogeneous enantioselective hydrogenation. Theory and practice. Springer, New York, p 118

35. Rykart R (1971) Bergkristall. Ott Verlag, Thun, p 70

36. Carter WC, Baram M, Drozdov M, Kaplan WD (2010) Scripta Mater 62:894 